\title{
A New Method for Evaluating the Bitterness of Medicines in Development Using a Taste Sensor and a Disintegration Testing Apparatus
}

\author{
Tsutomu Harada, ${ }^{*, a}$ Takahiro UchidA, ${ }^{b}$ Miyako YoshidA, ${ }^{b}$ Yoshikazu Kobayashi, ${ }^{c}$ \\ Ryuichi NARAZAKI, ${ }^{d}$ and Takayuki OHWAKI ${ }^{e}$ \\ a Planning \& Operations Section, Customer Joy Department, CEO office, Eisai Co., Ltd.; 5-5-5 Koishikawa, Bunkyo-ku, \\ Tokyo 112-8088, Japan: ${ }^{b}$ School of Pharmaceutical Sciences, Mukogawa Women's University; 11-68 Koshien 9-Bancho, \\ Nishinomiya, Hyogo 663-8197, Japan: ' Intelligent Sensor Technology, Inc.; 5-1-1 Onna, Atsugi, Kanagawa 243-0032, \\ Japan: ${ }^{d}$ Formulation Research, CMC Japan, Pharmaceutical Science and Technology CFU, Eisai Co., Ltd.; and ${ }^{e}$ Drug \\ Development Technology Center, Customer Joy Department, CEO Office, Eisai Co., Ltd.; 1 Kawashimatakehaya-machi, \\ Kakamigahara, Gifu 501-6195, Japan.
}

Received December 11, 2009; accepted May 14, 2010; published online May 28, 2010

The purpose of this study was to demonstrate the usefulness and wide applicability of a taste sensor and a new disintegration testing apparatus in the development and/or evaluation of orally disintegrating tablets (ODTs). In this paper, we described methods for the effective utilization of a taste sensor in the development of a new medicine. First we predicted the taste of propiverine hydrochloride, a model drug substance whose taste is unknown, using a taste sensor. Then we screened masking agents for their ability to suppress the bitterness of propiverine hydrochloride, and manufactured ODTs of propiverine hydrochloride with various masking agents. The tastes of these ODTs were then evaluated in chronological order by combining the taste sensor with the new disintegration testing apparatus, ODT-101, to resemble the oral cavity. As a result, we were able to evaluate the taste of propiverine hydrochloride and the effectiveness of various masking agents in ODTs. The result using this combination of taste sensor and ODT-101 shows good agreement with the results of human gustatory sensation testing, thus demonstrating the usefulness and applicability of the taste sensor and disintegration testing apparatus, ODT-101, in the development of new medicine.

Key words taste sensor; disintegration testing apparatus; propiverine hydrochloride; orally disintegrating tablet; taste masking agent; bitterness suppression

The purpose of this study was to demonstrate the usefulness and wide applicability of a taste sensor and a new disintegration testing apparatus in the development of new drug products. In recent years, orally disintegrating tablets, which are easy for children or elderly people to take, have become increasingly important in improving compliance. In the case of drug substances with unpleasant tastes, the evaluation of taste of the drug and the technology of taste masking seems to be so important in formulation design.

At the same time, intense international competition in the field of drug development has created a vital need for improvements in the efficiency of drug development methods. The early design of a simplified formulation, prior to the development of a final commercial product, has become an important strategy in the early drug development process, to allow clinical trials to start earlier and permit screening of new molecular entities. A simple solution or suspension of a drug substance is often the first choice for early testing, as this will have the highest bioavailability and produce the highest bodily exposure. The new chemical entity cannot be tasted, however, for safety reasons, so researchers must investigate its taste in other ways in order to allow a single-blind trial using the active material and a placebo.

There have been various studies on the quantitative evaluation of taste of medicines using a taste sensor. ${ }^{1-9)}$ Most previous experimental studies using the taste sensor have involved the quantitative evaluation of medicines with known tastes or bitterness suppression of a known product. ${ }^{10-24)}$ However, no research has yet been carried out to predict the taste of an unknown medicine. Taste sensors can measure only liquid samples. Therefore, researchers can only measure taste after dissolving solid or semi-solid samples in medium.
In the case of an orally disintegrating tablet (ODT), however, taste is evaluated in the process of dissolving the ODT in the saliva of the oral cavity, leaving a possibility that the measurement of the taste of an ODT solution is different from the measurement of taste during the process of ODT disintegration in the oral cavity.

In this paper, therefore, we describe methods for the effective utilization of a taste sensor and a new disintegration testing apparatus in the development of a new medicine. First we predicted the taste of propiverine hydrochloride, a model drug substance whose taste is unknown, using a taste sensor. Then we screened masking agents for their ability to suppress the unpleasant taste of propiverine hydrochloride, and manufactured ODTs of propiverine hydrochloride with various masking agents. The tastes of these ODTs were then evaluated in chronological order by combining the taste sensor with the new disintegration testing apparatus, ODT-101, to resemble the oral cavity.

\section{Experimental}

Materials Propiverine hydrochloride powder was purchased from Apogepha Arzneimittel GmbH (Dresden, Germany). Basic taste standards were prepared by Intelligent Sensor Technology, Inc. (Kanagawa, Japan). Quinine hydrochloride, ticlopidine hydrochloride and sucrose were purchased from Wako Pure Chemical Industries, Ltd. (Osaka, Japan). Eperizone hydrochloride, donepezil hydrochloride and azelastine hydrochloride were manufactured by Eisai Co., Ltd. (Tokyo, Japan); $\kappa$-, $t$-, and $\lambda$-carrageenan and agar were gifts from Ina Food Industry Co., Ltd. (Nagano, Japan). The carrageenans are a polysaccharide family built up from subunits consisting of two galactose rings that carry an electric charge of up to three unit charges derived from dissociated sulfate groups. The ideal structures indicate that the $\kappa-, t-$, and $\lambda$-carrageenans should be characterized by one, two, or three charge units, respectively, per subgroup. ${ }^{25)} \mathrm{LM}$ pectin was purchased from Sansho Co., Ltd. (Osaka, Japan); Ludiflash ${ }^{\circledR}$, a granulated product developed by BASF for production of ODTs by the direct tabletting 
Table 1. Lipids and Plasticizers Used for the Membranes of Sensors

\begin{tabular}{lll}
\hline \hline Sensor (target taste) & \multicolumn{1}{c}{ Lipid } & Plasticizer \\
\hline AAE (for umami) & 1) Trioctylmethylammomium chloride & Di- $n$-octylphenyl phosphonate \\
CT0 (for saltiness) & 2) Phosphoric acid di(2-ethylhexyl) ester & Di- $n$-octylphenyl phosphonate \\
CA0 (for sourness) & 1) Tetradodecyl ammonium bromide & Detyl alcohol $n$-octylphenyl phosphonate \\
& 2) Trioctylmethylammomium chloride & \\
AE1 (for astringency) & 2) Phosphoric acid di(2-ethylhexyl) ester & Di- $n$-octylphenyl phosphonate \\
C00 (for acidic bitterness) & 3) Oleic acid & 2-Nitro phenyloctyl ether \\
K523 (for basic bitterness) & Tetradodecyl ammonium bromide & 1) Bis(1-butylpentyl) adipate \\
& Phosphoric acid di(2-ethylhexyl) ester & 2) Tributyl $o$-acetylcitrate
\end{tabular}

Table 2. Compositions of Tested Samples

\begin{tabular}{ll}
\hline \hline \multicolumn{1}{c}{ Taste } & \multicolumn{1}{c}{ Sample } \\
\hline Reference solution & $30 \mathrm{~mm}$ potassium chloride $+0.3 \mathrm{~mm}$ tartaric acid \\
Saltiness & $270 \mathrm{~mm}$ sodium chloride \\
Sourness & $2.7 \mathrm{~mm}$ citric acid \\
Umami & $10 \mathrm{~mm}$ monosodium glutamate \\
Sweetness & $1 \mathrm{~m} \mathrm{sucrose}$ \\
Bitterness & $0.1 \mathrm{~mm}$ quinine hydrochloride \\
Astringency & $0.05 \%$ tannic acid \\
Unknown taste & $0.1 \mathrm{~mm}$ propiverine hydrochloride \\
\hline
\end{tabular}

method, and magnesium stearate (Mallinckrodt Pharmaceuticals, U.S.A.), were used for production of ODTs.

Sensor Measurement The taste-responding system SA402B (Taste Sensor) of Intelligent Sensor Technology, Ltd., Japan, was used to measure the electric potential of sample solutions. The electrode set is attached to a mechanically controlled robot arm. The detecting sensor part of the equipment consists of six electrodes composed of lipid/polymer membranes (channels). The lipids and plasticizers used for the membranes of the sensor are shown in Table 1. Each lipid was mixed in a test tube containing poly (vinyl chloride) and dioctylphenyl phosphonate as a plasticizer, dissolved in tetrahydrofuran, and dried on a glass plate at $30{ }^{\circ} \mathrm{C}$ to form a transparent thin film, almost $200 \mu \mathrm{m}$ thick. The electrodes consisted of a silver wire whose surface was plated with $\mathrm{Ag} / \mathrm{AgCl}$, with an internal cavity filled with $3.33 \mathrm{M}$ $\mathrm{KCl}$ solution. The difference between the electric potentials of the working electrode and the reference electrode was measured as the sensor output, by means of a high input impedance amplifier connected to a computer. Six sensors were used for the prediction of the taste of propiverine hydrochloride, only one of which, K523, was used for the evaluation of bitterness.

Fresh $30 \mathrm{~mm}$ potassium chloride solution containing $0.3 \mathrm{~mm}$ tartaric acid (corresponding to saliva) was used as the reference solution and also to rinse the electrodes after every measurement as described in previous articles. ${ }^{21)}$ The electrode is first dipped into the reference solution $(\mathrm{Vr})$ and then into the sample solution (Vs). The relative sensor output is represented as the difference $(\mathrm{Vs}-\mathrm{Vr})$ between the potentials of the sample and the reference solution. The difference $\left(\mathrm{Vr}^{\prime}-\mathrm{Vr}\right)$ between the potentials of the reference solution before and after sample measurement is defined as CPA (change of membrane potential caused by adsorption) and corresponds to aftertaste and bitterness. The measuring intervals were set at $30 \mathrm{~s}$, and the electrodes were rinsed after each measurement.

Prediction of Taste of Propiverine Hydrochloride The taste of propiverine aqueous solution was measured by the taste sensor in comparison with the five basic taste solutions (saltiness, sourness, umami, sweetness, bitterness) and astringency. The compositions of the sample tested and the basic taste solutions are shown in Table 2.

Human Sensory Evaluation of Bitterness Prior to the test, all volunteers were briefed in detail on the purpose of the test and gave informed consent. The sensory evaluations were performed with six well-trained volunteers according to a previously described method. ${ }^{26)}$ The standard quinine hydrochloride concentrations used were $0.01,0.03,0.10,0.30$, and $1.00 \mathrm{~mm}$ and the corresponding bitterness scores were defined as $0,1,2,3$, and 4 , respectively. Before testing, the volunteers were asked to keep the above-mentioned standard quinine solutions in their mouths, and were told the concen-
Table 3. Compositions of Solutes in $450 \mathrm{ml}$ Tested Samples

\begin{tabular}{cl}
\hline \hline No. & \multicolumn{1}{c}{ Composition of solutes in $450 \mathrm{ml}$ solution } \\
\hline 1 & $5 \mathrm{mg}$ propiverine hydrochloride (control) \\
2 & $5 \mathrm{mg}$ propiverine hydrochloride $+5 \mathrm{mg} \kappa$-carrageenan \\
3 & $5 \mathrm{mg}$ propiverine hydrochloride $+5 \mathrm{mg}$-carrageenan \\
4 & $5 \mathrm{mg}$ propiverine hydrochloride $+5 \mathrm{mg} \lambda$-carrageenan \\
5 & $5 \mathrm{mg}$ propiverine hydrochloride $+5 \mathrm{mg}$ agar \\
6 & $5 \mathrm{mg}$ propiverine hydrochloride $+5 \mathrm{mg}$ pectin \\
7 & $5 \mathrm{mg}$ propiverine hydrochloride $+45 \mathrm{~g}$ sucrose \\
\hline
\end{tabular}

Table 4. Compositions of Propiverine Hydrochloride ODTs

\begin{tabular}{|c|c|c|c|c|c|c|}
\hline Composition & Control & $\begin{array}{c}\text { Form. } \\
1\end{array}$ & $\begin{array}{c}\text { Form. } \\
2\end{array}$ & $\begin{array}{c}\text { Form. } \\
3\end{array}$ & $\begin{array}{c}\text { Form. } \\
4\end{array}$ & $\begin{array}{c}\text { Form. } \\
5\end{array}$ \\
\hline Propiverine (mg) & 5 & 5 & 5 & 5 & 5 & 5 \\
\hline Ludiflash $^{\circledR}(\mathrm{mg})$ & 193 & 188 & 188 & 188 & 188 & 188 \\
\hline Magnesium stearate (mg) & 2 & 2 & 2 & 2 & 2 & 2 \\
\hline$\kappa$-Carrageenan (mg) & & 5 & & & & \\
\hline l-Carrageenan (mg) & & & 5 & & & \\
\hline$\lambda$-Carrageenan (mg) & & & & 5 & & \\
\hline Agar (mg) & & & & & 5 & \\
\hline Pectin (mg) & & & & & & 5 \\
\hline Total (mg) & 200 & 200 & 200 & 200 & 200 & 200 \\
\hline
\end{tabular}

trations and bitterness scores of each solution. They were then asked to give the samples a bitterness score. All samples were kept in the mouth for $5 \mathrm{~s}$. After tasting each sample, subjects gargled well.

Evaluation of the Effects of Taste Masking Agents The ability of various polysaccharides and a sweetening agent to suppress the unpleasant taste of the propiverine was measured by the taste sensor by dissolving $5 \mathrm{mg}$ of propiverine hydrochloride in about $450 \mathrm{ml}$ of purified water. Each of $\kappa-, l-$, $\lambda$-carrageenan, pectin, agar, and sucrose was added to the propiverine solution as masking agent in the ratio shown in Table 3. These solutions were heated to $90^{\circ} \mathrm{C}$ for $30 \mathrm{~min}$, and then were cooled to room temperature. $\mathrm{KCl}$ was added to stabilize electrical conductivity for the measurement of the taste sensor; the $\mathrm{KCl}$ concentration was finally adjusted to $10 \mathrm{~mm}$ in $450 \mathrm{ml}$ solution.

Preparation of ODTs Compositions of propiverine hydrochloride ODTs are shown in Table 4. Sucrose was not used as a masking agent because the amount of sucrose required to suppress the bitterness of propiverine hydrochloride would be too much to allow the formulation of an ODT. The other polysaccharides were added to ODTs as powders. Magnesium stearate was employed as a lubricant, and lubrication was carried out using a tumbler mixer (Toyo Packing Co., Ltd.). Tabletting was carried out in Tab Flex ${ }^{\circledR}$ which was developed by the author and Okada Seiko Co., Ltd. as a device to evaluate the compression-molding characteristics of powders. ${ }^{27)}$

Measurement of the Taste of the ODT To measure the taste of the propiverine ODTs during the dissolution of the ODT, the disintegration testing apparatus ODT-101 (Toyama Sangyo Co., Ltd., Japan), developed by the authors, ${ }^{28-30)}$ was developed to resemble the oral cavity, and to be used together with the taste sensor. 
Figure 1 is an illustration of the core structure of the ODT-101. An ODT sample is placed on a stainless-steel porous plate. Weight (2) is provided to the shaft (1) which is capable of moving in the vertical direction and rotating. The liquid surface of the test medium $(450 \mathrm{ml}$ purified water) is automatically adjusted by a pump so that the water level is slightly below the lower face of the porous plate. The liquid temperature is set to $37^{\circ} \mathrm{C}$. In this study, a 10 -g weight was attached to a shaft and the shaft rotation rate was set at $25 \mathrm{rpm}$. When the measurement start button is pressed, the shaft goes down. The ODT is sandwiched between the rotating weight and the porous plate such that the load and shear force can be applied to the ODT. Simultaneously, the block (3) is immersed in the test medium and the water level of the test medium increases, such that the lower face of the ODT is in contact with the test medium. The ODT then absorbs the test medium by capillary suction, resulting in disintegration. The effects of load, shear, and wetting, reproduce the conditions in the oral cavity in which an ODT becomes wet with saliva and is lightly ground between the tongue and the roof of the mouth.

We measured the test medium $15 \mathrm{~s}$ after starting disintegration of an ODT and after full disintegration. In the 15 -s test, ODT-101 was stopped and the ODT removed from the test medium. Subsequently the test medium was filtered through mesh (No. 325) to produce the 15 -s sample. In the same way, ODT-101 testing was stopped immediately after full disintegration of the ODT, and the test medium filtered to produce the fully-disintegrated ODT

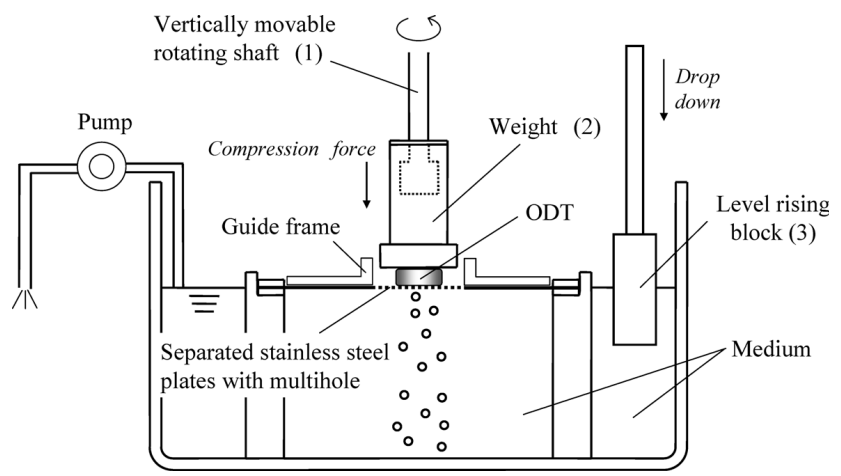

Fig. 1. Ilustration of the Core Structure of a New Disintegration Testing Apparatus ODT-101 sample.

Sensory Evaluation of the Taste of ODT The human gustatory sensation tests were performed with six well-trained volunteers. Prior to the test, all volunteers were given a detailed briefing on the purpose of the test and gave informed consent. They were asked to rinse their mouths out with water. The control ODT of propiverine was used as a standard. At first, volunteers allowed the control ODT to disintegrate in their mouths and awarded it a bitterness score of 5 . Then a sample was placed on the tongue and they were allowed to move the ODT with their tongues in the mouth. The swallowing of saliva was prohibited during the test. Immediately after the last noticeable granule or fragment had disintegrated, the volunteers rated the sample on an ascending bitterness scale of 0 to 5. After testing each sample, the volunteers gargled well.

\section{Results and Discussion}

Prediction of the Taste of Propiverine Hydrochloride First, the effect of propiverine aqueous solution on the responses of six sensors comprising different lipid membranes were compared with the six basic taste substances listed in Table 2. Figure 2 shows the sensor responses. The error bars represent standard deviations. Each sensor, except the umami taste sensor AAE and the acid taste sensor CAO, responded specifically to each basic taste substance, making it possible for the taste sensor to correctly identify each taste. Sensor K523 responds selectively to only a bitter taste. ${ }^{24)}$ Propiverine solution therefore seems to a have bitter taste, as K523 responded as much to the propiverine solution as the quinine solution. Principle component analysis was applied to the data, as the responses of the umami taste sensor AAE and the acid taste sensor CAO look similar. The results are shown in Fig. 3, which shows that principal component 1 (PC1) indicates astringency, because the plotted position of the tannic acid is farthest from the reference solution point. Similarly, PC2 indicates umami taste, PC3 indicates salty taste, and PC4 indicates bitter taste. Figure 3a shows that the umami taste of monosodium glutamate (MSG) and the sourness of (a) $\mathrm{AAE}$

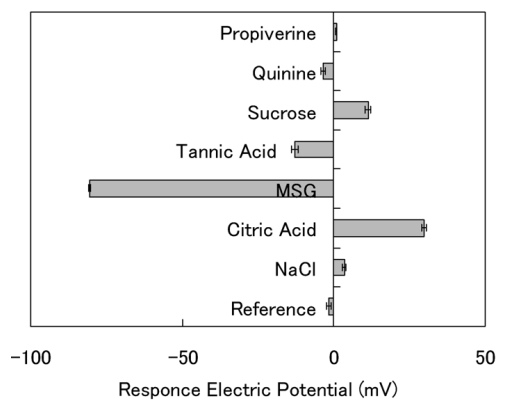

(d) CPA of AE1

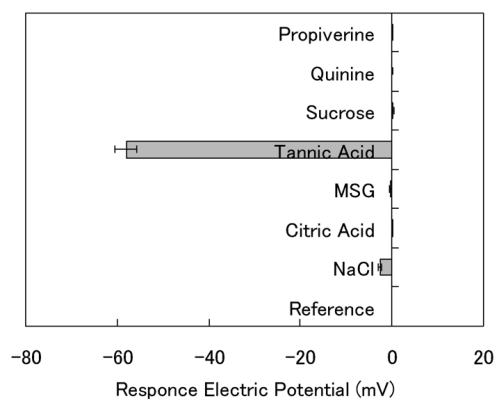

(b) CTO

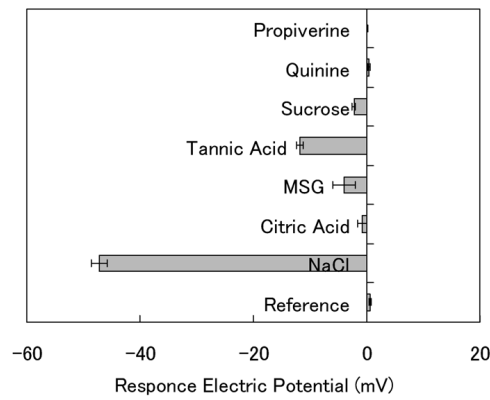

(e) CPA of $\mathrm{COO}$

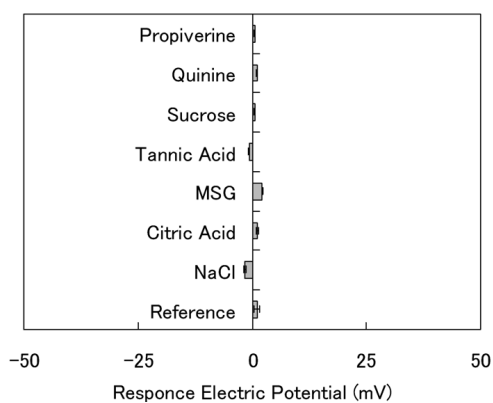

(c) CAO

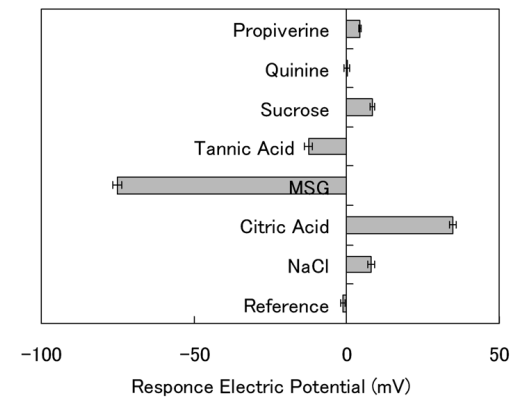

(f) CPA of K523

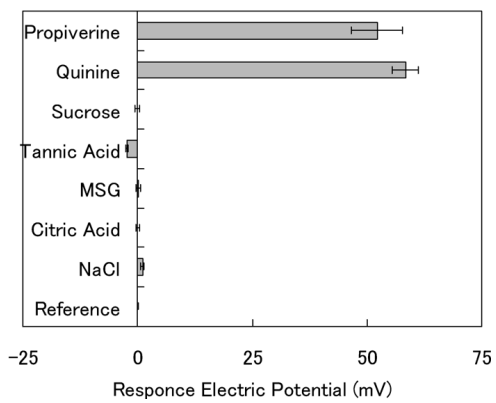

Fig. 2. Responses of Sensors Comprising Different Lipid Membranes to Six Basic Taste Substances and a Tested Drug Substance Values are mean \pm S.D. 
(a)

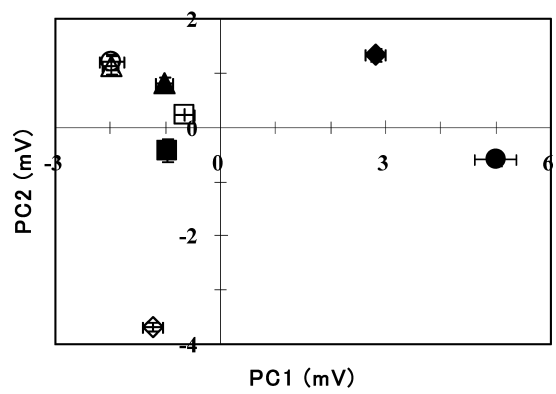

(b)

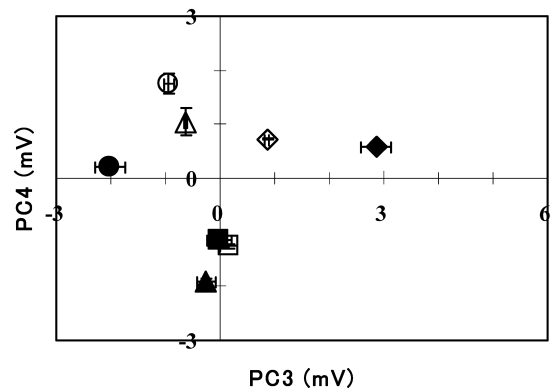

Fig. 3. Principal Component (PC) Analysis of Sensor Output Values for Basic Tastes and Tested Drug Substance

(a) PC1: contribution rate: $48.1 \%$, PC2: contribution rate: $20.8 \%$; (b) $\mathrm{PC} 3$ : contribution rate: $15.6 \%$, PC4: contribution rate: $12.6 \%$. Values are mean \pm S.D.; reference solution $(\square)$, tanninc acid $(\bullet), \operatorname{MSG}(\diamond), \mathrm{NaCl}(\diamond)$, quinine $\mathrm{HCl}(\bigcirc)$, propiverine $\mathrm{HCl}$ $(\triangle)$, citric acid $(\mathbf{\Delta})$, sucrose $(\square)$

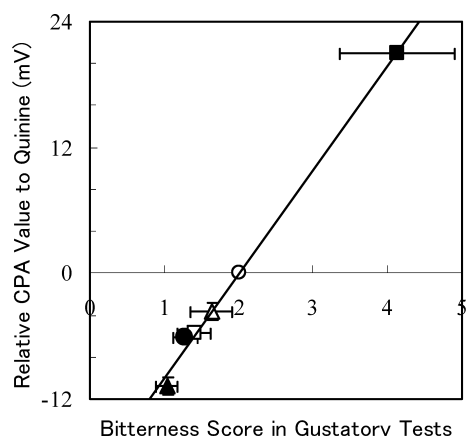

Fig. 4. The Correlation between the Relative CPA Value of Quinine Solution and the Bitterness Score Determined in Human Sensory Evaluation

Values are mean \pm S.D.; $Y=9.91 X-19.8, R=0.998$; quinine $\mathrm{HCl}(O)$, propiverine $\mathrm{HCl}(\triangle)$, donepezil $\mathrm{HCl}(\square)$, eperisone $\mathrm{HCl}(\bullet)$, ticlopidine $\mathrm{HCl}(\boldsymbol{\Delta})$, azelastine $\mathrm{HC}$ (缟

citric acid are readily identifiable as different tastes. Propiverine is confirmed to have a similar taste to quinine, as propiverine and quinine are plotted at almost the same point in the evaluation of PC1, PC2 and PC3. Meanwhile, Fig. 3b shows that quinine has a more bitter taste than propiverine according to PC4.

Practical prediction of drug bitterness is important in the drug development process. Figure 4 shows the correlation between the relative CPA value and the bitterness score determined in human sensory testing, when the propiverine solution was compared with a solution of a known bitter drug. The relative CPA value (vertical axis) of the bitterness sensor K523 represents the relative electric potential of the sample in relation to that of the quinine solution; a positive value indicates a more bitter taste than that of the quinine solution.

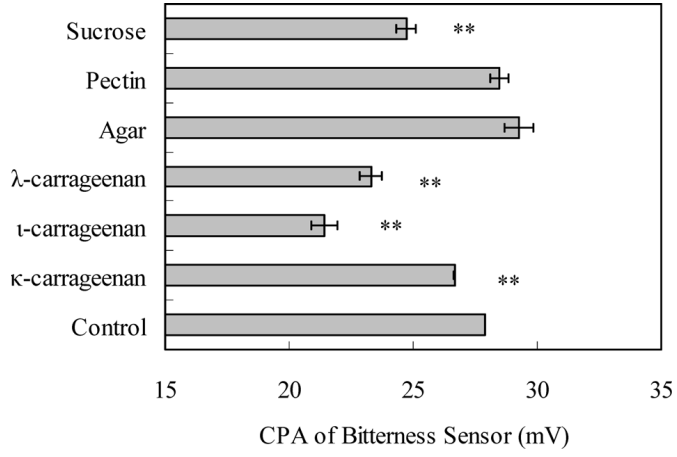

Fig. 5. Effects of Masking Agents on Propiverine Solution

Values are mean \pm S.D.; $* * p<0.01 v$ s. control.

From the relative CPA values, we could predict that the propiverine solution was a little less bitter than donepezil solution but not as bitter as quinine solution. When the results from the taste sensor evaluation are compared with those from human sensory testing, the two data sets are in close agreement.

In summary, the results of Figs. 2 and 3 suggest that propiverine hydrochloride has a bitter taste; the results of Fig. 4 predict the bitterness intensity of propiverine hydrochloride and show good agreement between the predictions of the taste sensor and gustatory sensation testing. Our results confirm that the taste sensor can predict the taste of a drug without human sensory testing and suggest that the taste sensor may be very useful in the formulation development of new chemical entities or hazardous drugs.

Evaluation of Bitterness Suppression of Propiverine by the Taste Sensor Figure 5 shows the effects of masking agents on the CPA values of the bitterness sensor K523 for propiverine aqueous solution. The greatest bitterness-suppressing effect was shown by $l$-carrageenan, followed by $\lambda$ carrageenan, and sucrose. $\kappa$-Carrageenan had very little suppression effect at the concentrations used, while agar and pectin had no effect on propiverine solution, even though they are also viscous polysaccharides. And, sucrose had negligibly-small suppression effect, because the concentration of sucrose was 9000 times higher than that of the other polysaccharides. The carrageenans seem to use the following suppression mechanisms: in aqueous solution carrageenans have negatively-charged sulfate groups, while propiverine is a cation. When carrageenan is added to propiverine solution, there is electrostatic interaction between them. In the oral cavity ionized propiverine binds to bitter taste receptors on the mucous membrane, thereby giving a bitter taste. The electrostatic interaction between propiverine and the carrageenan may decrease the contact between propiverine and the mucous membrane in the oral cavity. Agar could not suppress the bitter taste, because it is electroneutral. This fact indicates that just putting a viscous polysaccharide in the drug solution is not going to suppress the bitterness taste. The reason for the $l$-carrageenan being more effective than the $\kappa$ carrageenan can be explained by the number of sulfate groups, the $\kappa$-carrageenan having one sulfate group and the $l$-carrageenan having two per an idealized disaccharide repeating structure of the carrageenans. This theory does not, however, explain why the $l$-carrageenan suppresses bitterness more effectively than the $\lambda$-carrageenan, as $\lambda$-carrageenan 
(a)

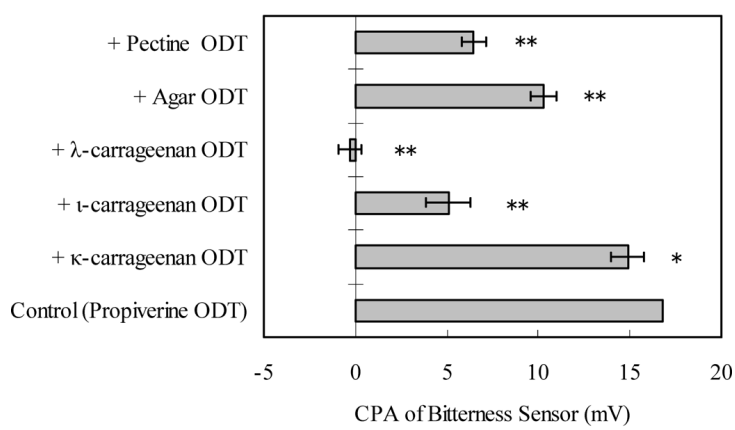

(b)

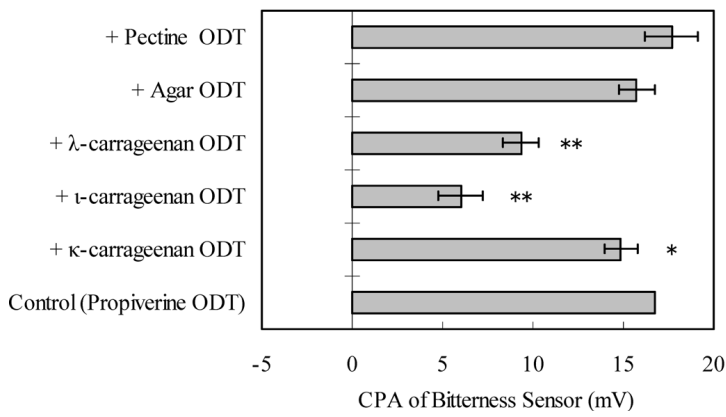

Fig. 6. (a) CPA of Bitterness Sensor of Propiverine ODT at $15 \mathrm{~s}$. (b) CPA of Bitterness Sensor of Propiverine ODT Soon after Full Disintegration

Values are mean \pm S.D.; $* * p<0.01, * p<0.05$ vs. control.

has three sulfate groups per subgroup. It is commonly considered that the $l$-carrageenan and the $\kappa$-carrageenan exist in helical form in aqueous solution, and the sulfate groups face outside. $^{31-34)}$ Therefore, the drug molecule may be sandwiched in between the sulfate groups of $l$ - or $\kappa$-carrageenan. In contrast, $\lambda$-carrageenan exists in a random coil form in aqueous solution. ${ }^{35)}$ The drug molecule interacting with the carrageenan in a random coil form can uncouple more easily than in the helical form. Therefore, $l$-carrageenan suppresses the bitter taste of a drug molecule more effectively than $\lambda$ carrageenan. While pectin has a carboxylic group, pectin in aqueous solution seems to be in the coil form. The drug-pectin interaction is weaker than the drug-carrageenan interaction.

Dynamic Evaluation of the Taste of Propiverine Hydrochloride ODT Figure 6a shows the CPA values of bitterness sensor K523 in the test medium $15 \mathrm{~s}$ after starting disintegration of the propiverine ODTs with masking agents. From this result, it was predicted that the greatest bitternesssuppressing agent would be $\lambda$-carrageenan, followed by $l$ carrageenan, pectin, agar, and $\kappa$-carrageenan. It should be noted that polysaccharide powders, rather than dissolved polysaccharides, can suppress the bitter taste of drugs. Figure $6 a$ differs from Fig. 5 in the following respects: (1) $\lambda$-carrageenan suppresses the bitter taste more effectively than $l$ carrageenan, and (2) pectin and agar both suppress the bitter taste of drug in ODT. The former result (1) is attributed to the rate of dissolution, with $\lambda$-carrageenan acting more rapidly than $l$-carrageenan because $\lambda$-carrageenan dissolves in water more rapidly. The carrageenan never suppress the bitter taste unless it dissolves. The latter result (2) is explained by the disintegration rate. The disintegration times of the pro-
Table 5. Disintegration Time of Propiverine Hydrochloride ODTs

\begin{tabular}{ccc}
\hline \hline Form. & Masking agent & Disintegration time (s) \\
\hline Control & - & 13.97 \\
1 & l-Carrageenan & 14.04 \\
2 & $\lambda$-Carrageenan & 29.86 \\
3 & Agar & 25.02 \\
4 & Pectin & 32.71 \\
5 & & 34.23 \\
\hline
\end{tabular}

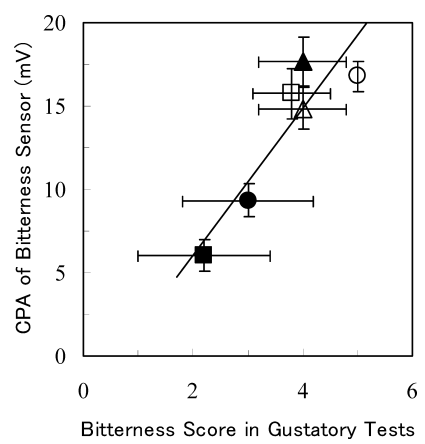

Fig. 7. The Correlation between the CPA Value of Bitterness Sensor and the Bitterness Score Determined in Human Sensory Evaluation Soon after Full Disintegration of the ODTs

Values are mean \pm S.D.; $Y=4.39 X-2.70, R=0.907, p<0.005$; control $(\bigcirc), \kappa$-carrageenan $(\triangle), l$-carrageenan $(\boldsymbol{\square}), \lambda$-carrageenan $(\bullet)$, agar $(\square)$, pectine $(\boldsymbol{\Delta})$.

piverine ODTs with masking agents are shown in Table 5. ODTs containing pectin or agar disintegrated more slowly than the ODT without these viscous polysaccharides. Therefore, the dissolution of propiverine from an ODT containing pectin or agar was lower by $15 \mathrm{~s}$ after the start of disintegration.

Figure $6 \mathrm{~b}$ shows the CPA values of the bitterness sensor $\mathrm{K} 523$ in the test medium after full disintegration of the ODTs. From this result, it was predicted that the greatest effect on bitterness suppression would be that of $l$-carrageenan, followed by $\lambda$-carrageenan and $\kappa$-carrageenan. However, pectin and agar had no effect of bitterness suppression. This result agrees with that obtained from Fig. 5.

Figure 7 shows the correlation between the CPA value of the bitterness sensor and the bitterness score in gustatory tests soon after full disintegration of propiverine ODTs. The regression formula is written in the figure. The correlation coefficient $(R)$ is 0.907 . From this result, the predicted bitterness of ODTs using the taste sensor and the disintegration testing apparatus ODT-101 were found to be very similar to the bitterness scores in gustatory tests. However, the bitterness scores of gustatory sensation varied widely, compared with the CPA values of the bitterness sensor. It may be concluded that this combination of taste sensor and ODT-101 is more correct for measuring the taste of ODTs than the human sensory evaluation. In addition, it can be seen from the viewpoint of ensuring the safety of volunteers that the sensory evaluation should be replaced by the sensor measurement using the taste sensor and the ODT-101.

The results from Figs. $6 a$ and $b$ indicate that the taste of the propiverine hydrochloride ODT is affected by the disintegration rate of ODT and the solubility rate of masking agent, and the taste changes as the ODT disintegrates. The relation- 


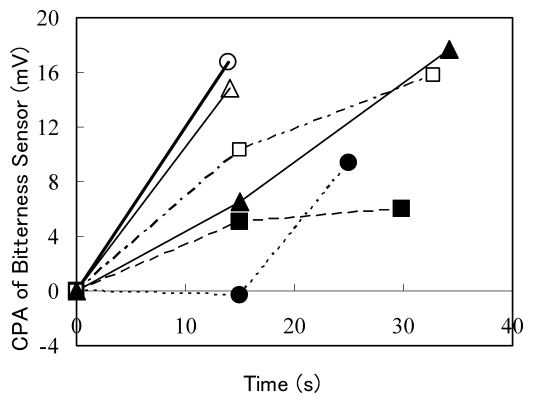

Fig. 8. Relationship between CPA of Bitterness Sensor and Disintegration Time of Propiverine ODT

Control $(\bigcirc), \kappa$-carrageenan $(\triangle), l$-carrageenan $(\square), \lambda$-carrageenan $(\bullet)$, agar $(\square)$, pectine $(\mathbf{\Delta})$.

ship between the CPA of the bitterness sensor and the disintegration time of the propiverine ODT is shown in Fig. 8. From the figure it may be deduced that the ODT containing $\lambda$-carrageenan can fully suppress bitter taste of the propiverine up to $15 \mathrm{~s}$ after starting disintegration of the propiverine ODTs. This result indicates that $\lambda$-carrageenan is the best masking agent if the ODT is taken with a glass of plain water. On the other hand, $l$-carrageenan suppresses the bitter taste by around $35 \%$ even after full disintegration of the propiverine ODT. Therefore, $l$-carrageenan is the best masking agent if the propiverine ODT is taken without water.

\section{Conclusions}

Methods for the effective utilization of a taste sensor in the development of a new medicine are presented. The taste of propiverine hydrochloride, a model drug substance whose taste is unknown, could be predicted using a taste sensor. Good agreement between the predictions of the taste sensor and gustatory sensation testing has been shown. Our results confirm that the taste sensor can predict the taste of a drug substance without human sensory testing.

Next, the effect of masking agents for suppressing the bitterness of propiverine hydrochloride could be predicted by the taste sensor, with the greatest bitterness-suppressing effect shown by $l$-carrageenan, followed by $\lambda$-carrageenan.

Finally, we evaluated the tastes of propiverine ODTs containing various polysaccharides in chronological order, by using the taste sensor and the new disintegration testing apparatus ODT-101 in combination. The bitter taste of propiverine in the ODT containing $\lambda$-carrageenan was fully suppressed for up to $15 \mathrm{~s}$ after the start of disintegration of the ODT. However, $l$-carrageenan was more effective than $\lambda$ carrageenan in suppressing bitterness after full disintegration of the ODT. The taste of the propiverine hydrochloride ODT changed as the ODT disintegrated because the taste was determined by balance between disintegration rate of ODT and solubility rate of masking agent. Just measuring taste after dissolving ODT in medium is not going to evaluate in the process of disintegrating the ODT in the oral cavity. The result using this combination of taste sensor and the new disintegration testing apparatus showed good agreement with the results of human gustatory sensation testing, thus demonstrating the usefulness and wide applicability of this combination in the development of ODTs.

\section{References}

1) Uchida T., Miyanaga Y., Tanaka H., Wada K., Kurosaki S., Ohki T., Yoshida M., Matsuyama K., Chem. Pharm. Bull., 48, 1843-1845 (2000).

2) Uchida T., Kobayashi Y., Miyanaga Y., Toukubo R., Ikezaki H., Taniguchi A., Nishikata M., Matsuyama K., Chem. Pharm. Bull., 49, 1336-1339 (2001).

3) Miyanaga Y., Tanigake A., Nakamura T., Kobayashi Y., Ikezaki H., Taniguchi A., Matsuyama K., Uchida T., Int. J. Pharm., 248, $207-$ 218 (2002)

4) Uchida T., Tanigake A., Miyanaga Y., Matsuyama K., Kunitomo M., Kobayashi Y., Ikezaki H., Taniguchi A., J. Pharm. Pharmacol., 55, 1479-1485 (2003).

5) Ishizaka T., Miyanaga Y., Mukai J., Asaka K., Nakai Y., Tsuji E., Uchida T., Chem. Pharm. Bull., 52, 943-948 (2004).

6) Anand V., Kataria M., Kukkar V., Saharan V., Choudhury K. P., Drug Discov. Today, 12, 257-265 (2007).

7) Kataoka M., Tokuyama E., Miyanaga Y., Uchida T., Int. J. Pharm., 351, 36-44 (2008)

8) Lorenz K. J., Reo P. J., Hendl O., Worthington H. J., Petrossian D. V., Int. J. Pharm., 367, 65-72 (2009).

9) Woertz K., Tissen C., Kleinebudde P., Breitkreutz J., J. Pharm. Biomed. Anal., 51, 497-506 (2010)

10) Takagi S., Toko K., Wada K., Yamada H., Toyoshima K., J. Pharm. Sci., 87, 552-555 (1998).

11) Takagi S., Toko K., Wada K., Ohki T., J. Pharm. Sci., 90, 2042-2048 (2001).

12) Nakamura T., Tanigake A., Miyanaga Y., Ogawa T., Akiyoshi T., Matsuyama K., Uchida T., Chem. Pharm. Bull., 50, 1589-1593 (2002).

13) Miyanaga Y., Kobayashi Y., Ikezaki H., Taniguchi A., Uchida T., Sensors Mater., 14, 455-465 (2002).

14) Hashimoto Y., Tanaka M., Kishimoto H., Shiozawa H., Hasegawa K., Matsuyama K., Uchida T., J. Pharm. Pharmacol., 54, 1323-1328 (2002).

15) Miyanaga Y., Inoue N., Ohnishi A., Fujisawa E., Yamaguchi M. Uchida T., Pharm. Res., 20, 1932-1938 (2003).

16) Ogawa T., Nakamura T., Tsuji E., Miyanaga Y., Nakagawa H., Hirabayashi H., Uchida T., Chem. Pharm. Bull., 52, 172-177 (2004).

17) Miyanaga Y., Mukai J., Mukai T., Odomi M., Uchida T., Chem. Pharm. Bull., 52, 490-493 (2004).

18) Zheng Y. J., Keeney P. M., Int. J. Pharm., 310, $118-124$ (2006).

19) Tsuji E., Uchida T., Fukui A., Fujii R., Sunada H., Chem. Pharm. Bull., 54, 310-314 (2006).

20) Tokuyama E., Shibasaki T., Kawabe H., Mukai J., Okada S., Uchida T., Chem. Pharm. Bull., 54, 1288-1292 (2006).

21) Ishizaka T., Okada S., Takemoto E., Tokuyama E., Tsuji E., Mukai J., Uchida T., Chem. Pharm. Bull., 55, 1452-1457 (2007).

22) Hashimoto Y., Matsunaga C., Tokuyama E., Tsuji E., Uchida T., Okada H., Chem. Pharm. Bull., 55, 739-746 (2007).

23) Agresti C., Tu Z., Ng C., Yang Y., Liang F. J., Eur. J. Pharm. Biopharm., 70, 226-233 (2008).

24) Kobayashi Y., Hamada H., Yamaguchi Y., Ikezaki H., Toko K., IEEJ Trans., 4, 710-719 (2009).

25) Lelham C. N., Sundelöf O. L., Int. J. Pharm., 115, 103-111 (1995).

26) Katsuragi Y., Mitsui Y., Umeda T., Otsuji K., Yamasawa S., Kurihara K., Pharm. Res., 14, 720-724 (1997).

27) Harada T., Nakata H., PCT WO 03/074263.

28) Narazaki R., Harada T., Takami N., Kato Y., Ohwaki T., Chem. Pharm Bull., 52, 704-707 (2004).

29) Harada T., Narazaki R., Nagira S., Ohwaki T., Aoki S., Iwamoto K., Chem. Pharm. Bull., 54, 1072-1075 (2006).

30) Harada T., Narazaki R., Ohwaki T., Uchida T., J. Drug Del. Sci. Tech., (2010), in press.

31) Anderson N. S., Campbell J. W., Harding M. M., Rees D. A., Samuel J. W. B., J. Mol. Biol., 45, 85-99 (1969).

32) Morris E. R., Rees D. A., Robinson G. O., J. Mol. Biol., 138, 349 362 (1980).

33) Ikeda S., Morris V. J., Nishinari K., Biomacromolecules, 2, 13311337 (2001).

34) Yuguchi Y., Thuy T. T. T., Urakawa H., Kajiwara K., Food Hydrocolloids, 16, 512-522 (2002).

35) De Ruiter G. A., Rudolph B., Trends Food Sci. Tech., 8, 389-395 (1997). 